\title{
Density dependence of the stress relaxation function of a simple fluid
}

\author{
Remco Hartkamp* \\ Multi Scale Mechanics, MESA + Institute for Nanotechnology, University of Twente, P.O. Box 217, 7500 AE Enschede, The Netherlands
}

Peter J. Daivis ${ }^{\dagger}$

School of Applied Sciences, RMIT University, GPO Box 2476, Melbourne, Victoria 3001, Australia

\author{
B. D. Todd ${ }^{\ddagger}$ \\ Mathematics Discipline, Faculty of Engineering and Industrial Sciences and Centre for Molecular Simulation, \\ Swinburne University of Technology, Hawthorn, Victoria 3122, Australia
}

(Received 12 September 2012; published 25 March 2013)

\begin{abstract}
We present accurate molecular dynamics calculations of the shear stress relaxation modulus of a simple atomic fluid over a wide range of densities. The high accuracy of the data enables us to study changes in the functional form of the shear relaxation modulus, and the properties that are derived from it, as the density is increased from the ideal gas limit to the upper limit of the fluid range. We show that the shear relaxation modulus of a dilute atomic fluid can be accurately described with a simple functional form consisting of a Gaussian plus a single exponential, whereas the dense fluid exhibits a more complicated relaxation function. The infinite-frequency shear modulus, the zero-shear viscosity, and the zero-shear first normal stress coefficient are calculated from the stress autocorrelation function. The ratio of the first normal stress coefficient to the viscosity is used to calculate the viscoelastic relaxation time. While the viscosity and the infinite-frequency shear modulus both increase monotonically with increasing density, the first normal stress coefficient and the viscoelastic relaxation time both decrease to a minimum at intermediate densities before increasing again. Our results for the viscosity and the first normal stress coefficient in the low-density limit both agree well with the predictions of kinetic theory.
\end{abstract}

DOI: 10.1103/PhysRevE.87.032155

PACS number(s): 66.20.Cy, 61.20.Lc

\section{INTRODUCTION}

At sufficiently high frequencies or shear rates, even simple atomic fluids are known to exhibit non-Newtonian phenomena, such as viscoelasticity, shear thinning, shear dilatancy, and normal stress differences [1]. Linear transport coefficients, like the zero-shear rate viscosity, characterize the Newtonian regime, while the shear-rate dependent viscosity and the first and second normal stress coefficients characterize the steady-state non-Newtonian behavior at higher shear rate. Similarly, the Newtonian viscosity can be seen as the lowfrequency limit of the complex frequency dependent viscosity that characterizes the frequency domain viscoelastic response. The stress relaxation function is an important property from which we can calculate these material constants. Its behavior as the density is increased from the ideal gas limit to the dense fluid is of great interest, not only because of its importance in studies of transport phenomena, but also in relation to the onset of the glass transition and pretransitional signatures of solidification [2]. It is also desirable to determine whether the stress relaxation function can be modeled with simple functional forms over the entire fluid density range. Although the functional form of the stress relaxation function for several simple liquids has been studied before at various temperatures and densities, there have been few comprehensive and systematic studies of its density dependence for a single system. A notable exception is the work by Heyes et al. [3,4], whose

\footnotetext{
*r.m.hartkamp@utwente.nl

†peter.daivis@rmit.edu.au

†btodd@swin.edu.au
}

main purpose was to study the behavior of the stress relaxation function for atomic fluids with steeply repulsive soft sphere potentials as they approach the hard-sphere limit.

Most studies of the viscoelastic properties of simple fluids have concentrated on the viscosity, the infinite-frequency shear modulus, and the Maxwell relaxation time. The first normal stress coefficient has received much less attention because it is more difficult to compute. The viscosity is usually calculated using equilibrium molecular dynamics (EMD) simulations and the Green-Kubo [5,6] relation that gives the viscosity in terms of the integral of the stress autocorrelation function (SACF). The shear relaxation function (often known as the shear relaxation modulus in the rheology literature) is directly proportional to the SACF.

The viscoelastic relaxation of simple fluids has been studied by a number of authors [2,7-12]. Mountain and Zwanzig [9] studied the density dependence of the Maxwell relaxation time of a supercritical Lennard-Jones fluid. They found a decrease in relaxation time with increasing density up to the maximum density studied, $\rho=0.7$. More recently, Keshavarzi et al. [11] studied the density and temperature dependence of the infinitefrequency shear modulus and the Maxwell relaxation time for purely repulsive soft sphere and Lennard-Jones fluids. They calculated the infinite-frequency shear modulus numerically from the equilibrium radial distribution function, using the relation derived by Zwanzig and Mountain [13]. A minimum relaxation time was again found around a reduced density of $\rho \approx 0.7$, independent of the temperature. The authors argued that this is a transition point, below which kinetic momentum transport dominates and above which the finite memory of the fluid dominates the flow behavior. 
Little computational work has been done on calculating the zero-shear rate normal stress coefficients [14], but measurements of these quantities are common in rheological experiments [15-17]. Loose and Hess [18] computed the nonequilibrium shear viscosity and normal stress coefficients for a moderately dense Lennard-Jones gas $(\rho=0.1, T=2.75)$ over a range of shear rates by homogeneous nonequilibrium molecular dynamics (NEMD). Their data show large statistical uncertainty for low strain rates, but their results for the shear rate dependent viscosity and the normal stress coefficients agree well with kinetic theory as shown in Ref. [19]. Although their system was a gas of moderate density, its behavior was well approximated by the ideal gas model because the state point chosen was close to the Boyle point, where the effects of attractive and repulsive interactions approximately cancel each other.

Coleman and Markovitz [20] derived an expression for the zero-shear rate first normal stress coefficient in terms of the linear stress relaxation modulus from a continuum theory of nonlinear viscoelasticity. Their expression is well known and verified experimentally in polymer rheology [21]. Calculation of the first normal stress coefficient using their method with EMD simulations is computationally expensive, even with modern computers, because it requires stress autocorrelation function data of extremely high precision. Recently, Daivis [14,22] used the Coleman-Markovitz method to calculate the zero-shear normal stress coefficient of a simple liquid. $\mathrm{He}$ also calculated the zero-shear viscosity and first normal stress coefficient using NEMD by extrapolating the shear-rate dependent viscosity and first normal stress coefficient to zero-shear rate, for a range of densities. While the equilibrium and nonequilibrium calculations of the viscosity agreed in the limit of zero-shear rate, the values of the first normal stress coefficient did not. A detailed understanding of this discrepancy is still lacking, but it is clear that the choice of thermostat has a strong influence on the values of nonlinear rheological properties calculated in NEMD simulations [22].

The shear relaxation function completely determines the linear viscoelastic shear response of a fluid. Enskog kinetic theory predicts that at low density, the stress autocorrelation function for a hard-sphere fluid should be a delta function at zero time due to the impulsive nature of hard-sphere forces, followed by an approximately single exponential decay [12]. The delta function results in an infinite value of the infinite-frequency shear modulus for the hard-sphere fluid at all densities, whereas the viscosity remains finite. In this case, the Maxwell relaxation time is zero, which would seem to imply that the stress relaxes to its steady-state value instantaneously after the onset of steady shear and that viscous flow of a hard-sphere fluid is perfectly inelastic. However, this is clearly not the case, because the kinetic part of the hard-sphere stress relaxation function has a nonzero relaxation time, and the overall relaxation of the SACF does not take place instantaneously. In this sense, the Maxwell time is a misleading measure of viscoelastic relaxation time. An alternative definition of the viscoelastic relaxation time, based on the ratio of the first normal stress coefficient to the viscosity, does not exhibit this anomaly.

The SACFs found in molecular dynamics simulations of hard-sphere fluids agree with the Enskog prediction at low density, where the kinetic part of the pressure tensor is dominant and the configurational part is negligible. Generalized kinetic theory [23] and an early mode coupling theory (MCT) calculation [24] both predict that the tail of the SACF should decay as $t^{-3 / 2}$ due to hydrodynamic effects that only contribute to the kinetic part of the stress autocorrelation function. However, the predicted hydrodynamic long-time tail in the stress autocorrelation function has only ever been (tentatively) observed by isolating the kinetic part of the stress autocorrelation function [25]. The total stress autocorrelation function also includes a configurational part and a cross term. Both of these exhibit long-time tails of amplitude much greater than that predicted by the conventional MCT [25]. Extended MCT [26-28] provides a much better prediction of the SACF behavior at moderate to high density. It predicts that the configurational and cross terms of the SACF decay as approximately $t^{-3 / 2}$ at intermediate times, but more rapidly at very long times. The amplitude of the intermediate time contribution is enhanced compared to the hydrodynamic tail predicted by kinetic theory and simple MCT, and its amplitude grows with increasing density. The existence of this part of the stress relaxation function, known as the "molasses tail" to distinguish it from the hydrodynamic long-time tail, is linked to structural relaxation [2,7] and the glass transition [29]. Although the extended MCT provides predictions of the stress relaxation function that agree with the results of molecular dynamics simulations of hard-sphere fluids, it does not result in a definite functional form that can easily be used to describe the whole relaxation function.

In this paper, we present results and reasonable empirically derived fits for the shear relaxation modulus of a simple atomic fluid over a wide range of densities. The onset of the molasses tail at high densities is shown to have an influence on the relaxation time and on the zero-shear normal stress coefficient of the fluid, whereas properties at low densities can be predicted well with kinetic theory. Finally, we will demonstrate the ability of the linear stress relaxation function obtained by EMD to predict a stress relaxation curve obtained from simulations of a sheared fluid relaxing to equilibrium.

This paper is organized as follows: Section II gives an introduction to viscometric functions and how to calculate them. The simulation details are given in Sec. III. Results are presented and discussed in Sec. IV. Finally, in Sec. V, our observations are summarized.

\section{THEORY}

The rheological behavior of a nonequilibrium fluid can be described by shear-rate dependent viscometric functions-for example, shear viscosity

$$
\eta(\dot{\gamma})=-\frac{P_{y x}}{\dot{\gamma}}
$$

and the first and second normal stress coefficients [30]

$$
\begin{aligned}
& \Psi_{1}(\dot{\gamma})=\frac{\mathcal{N}_{1}}{\dot{\gamma}^{2}}=\frac{P_{y y}-P_{x x}}{\dot{\gamma}^{2}}, \\
& \Psi_{2}(\dot{\gamma})=\frac{\mathcal{N}_{2}}{\dot{\gamma}^{2}}=\frac{P_{z z}-P_{y y}}{\dot{\gamma}^{2}},
\end{aligned}
$$


where $\mathbf{P}$ denotes the pressure tensor, $\mathcal{N}_{1}$ and $\mathcal{N}_{2}$ the first and second normal stress differences, and $\dot{\gamma}$ the shear rate, with the flow in the $x$ direction and the gradient of the streaming velocity in $y$. In the limit of zero-shear rate, the viscosity converges to the zero-shear viscosity

$$
\eta_{0}=\lim _{\dot{\gamma} \rightarrow 0} \eta(\dot{\gamma}),
$$

and the normal stress coefficients can approach values (typically nonzero) called the zero-shear normal stress coefficients

$$
\Psi_{\alpha, 0}=\lim _{\dot{\gamma} \rightarrow 0} \Psi_{\alpha}(\dot{\gamma}), \quad \alpha=1,2 .
$$

The second normal stress coefficient $\Psi_{2}$ is far less often studied than $\Psi_{1}$, because it is often much smaller and thus more difficult to measure accurately in the case of polymeric liquids [21]. It is also more difficult to obtain computationally, because there is currently no known method of obtaining it from an equilibrium molecular dynamics simulation. In the present study, we will focus on the first normal stress coefficient.

The zero-shear rate first normal stress coefficient can be related to the Deborah number De by

$$
\mathrm{De}=\frac{\Psi_{1,0}}{2 \eta_{0}} \sqrt{\mathrm{II} / 2}
$$

where $\eta_{0}$ is the Newtonian (zero-shear) viscosity and II is the second scalar invariant of the strain rate tensor $\mathbf{S}=\nabla \mathbf{u}+$ $(\nabla \mathbf{u})^{T}$. For shear flow, this expression simplifies to De $=\dot{\gamma} \tau$, where $\tau=\Psi_{1,0} /\left(2 \eta_{0}\right)$ is a relaxation time of the fluid.

Rather than extracting the viscometric quantities from nonequilibrium simulations and taking the zero-shear limit, the first normal stress coefficient can be calculated by evaluating an expression in equilibrium, derived by Coleman and Markovitz [20],

$$
\Psi_{1,0}=2 \int_{0}^{\infty} t G(t) d t
$$

where $G(t)$ is the (shear) stress relaxation modulus of a viscoelastic fluid, which is defined as the stress-strain ratio at a constant deformation rate. For an isotropic system, the stress relaxation modulus can be calculated as [14]

$$
G(t)=\frac{V}{10 k_{\mathrm{B}} T}\left\langle\mathbf{P}^{0 s}(t): \mathbf{P}^{0 s}(0)\right\rangle,
$$

where $V$ is the system volume, $k_{\mathrm{B}}$ Boltzmann's constant, $T$ the temperature, and the superscript " $0 s$ " denotes a traceless and symmetric tensor, which contains 5 independently fluctuating quantities. While this formulation is identical to the shear stress autocorrelation function that is commonly used as the susceptibility of the Green-Kubo shear viscosity integral, the tensorial approach is preferred for its enhanced statistics [31]. The pressure tensor is calculated from

$$
\mathbf{P}=\frac{1}{V} \sum_{i=1}^{N}\left(m_{i} \mathbf{v}_{i} \mathbf{v}_{i}-\frac{1}{2} \sum_{j \neq i} \frac{\mathbf{r}_{i j} \mathbf{r}_{i j}}{r} U^{\prime}(r)\right),
$$

where $N$ is the number of atoms in the system, $\mathbf{v}_{i}$ is the peculiar velocity vector, $\mathbf{r}_{i j}=\mathbf{r}_{i}-\mathbf{r}_{j}, r=\left|\mathbf{r}_{i j}\right|$, and $U^{\prime}$ is the derivative of the potential energy function. The first term on the righthand side is the kinetic part of the pressure tensor, whereas the second term is the configurational part.

The SACF of the traceless symmetric pressure tensor for a dense fluid is known to depend mostly on the configurational part [32] and thus depends strongly on the interaction potential. Heyes [33] calculated the dependence of several properties on the exponent in strongly repulsive potentials. He found that the infinite-frequency shear modulus increased approximately linearly with the exponent of the potential. Powles and Heyes [3] showed that in the hard-sphere limit (i.e., for steeply repulsive potentials), the shear stress and pressure correlation functions become simple analytic functions of the temperature and density. Furthermore, Brańka and Heyes [4] showed how the stress autocorrelation function depends on the exponent in strongly repulsive potentials. They showed that the decay time of the correlation function scales with the exponent of the interaction potential.

Viscoelastic fluids show a combination of elastic (solidlike) and viscous (liquid-like) stress response when a deformation is applied. The elastic response is proportional to the strain $\gamma$, with the elastic modulus $G$ as the proportionality constant. The shear viscosity $\eta$ is the proportionality constant relating the shear stress to the strain rate $\dot{\gamma}$. When a viscoelastic fluid is perturbed, it initially responds only elastically. The lag of the viscous response is related to the relaxation mechanism in viscoelastic fluids. In a steady-state (zero-frequency perturbation) flow, the elastic energy is stored during the approach to steady state. The real part of the shear modulus is zero for a fluid in the zero-frequency limit $[34,35]$. On the other hand, when a fluid is perturbed at very high frequencies, the elastic constant has a nonzero value. The infinite-frequency shear modulus $G(\omega \rightarrow \infty) \equiv G^{\infty}$ is a common measure for the elastic part of the response. This value corresponds to the initial value of the stress autocorrelation function $G^{\infty}=$ $G(t=0)=V\left\langle\mathbf{P}^{0 s}(0): \mathbf{P}^{0 s}(0)\right\rangle /\left(10 k_{\mathrm{B}} T\right)[13,36,37]$.

Similarly to the first normal stress coefficient [Eq. (7)], the zero-shear viscosity can be calculated from the stress relaxation modulus

$$
\eta_{0}=\int_{0}^{\infty} G(t) d t
$$

which is the Green-Kubo relation for shear viscosity. Using Eqs. (7) and (10), the viscous relaxation time is given by

$$
\tau=\frac{\Psi_{1,0}}{2 \eta_{0}}=\frac{\int_{0}^{\infty} t G(t) d t}{\int_{0}^{\infty} G(t) d t} .
$$

This expression makes no assumptions about the properties of the fluid or the shape of the relaxation function, apart from convergence of the relevant integrals. This expression differs from the definition of the Maxwell relaxation time, $\tau_{M}=\eta / G_{\infty}$, which can be written as

$$
\tau_{M}=\frac{\int_{0}^{\infty} G(t) d t}{G(0)} .
$$

In fact, a hierarchy of relaxation times can be defined, each one differing in the order of $t$ of the numerator and denominator, with the ratio remaining of order $t$. The Maxwell time can therefore be seen as a zeroth-order relaxation time, and the 
one defined in Eq. (11) can be seen as a first-order relaxation time. The highest order relaxation time that can be evaluated depends on the convergence properties of the integrals. For an exponential stress relaxation function, the relaxation times of all orders exist, and they are all equal to the Maxwell time. However, not all stress relaxation functions are exponential in time.

The stress relaxation time can also be directly measured from nonequilibrium simulations in which the fluid relaxes from a nonequilibrium steady state after the driving field is switched off. Gao and Weiner [38] observed that the relaxation of a simple fluid could be described using only 2 exponential modes. The authors performed their simulations without using a strictly homogeneous algorithm, such as the SLLOD equations of motion $[39,40]$. However, their system is stiff to ensure that the propagation of information is fast, resulting in only a small inaccuracy in the transient response. Picu and Weiner [8] showed, for a simple fluid under planar elongational flow, that the first relaxation mode corresponds to the distribution of nearest neighbors, while the second mode is related to the relaxation of anisotropy of the number density distribution.

Linear viscoelastic theory can be used to predict the response or shear stress after sudden cessation of steadystate shear flow (constant nonzero shear rate for $t<0$ ). The instantaneous shear stress of a viscoelastic fluid after the cessation of steady shear depends on the full history of the flow. The most general linear constitutive relation is

$$
P_{y x}(t)=-\int_{-\infty}^{t} G\left(t-t^{\prime}\right) \dot{\gamma}\left(t^{\prime}\right) d t^{\prime} .
$$

To calculate the shear stress after the cessation of steady shear, we can apply a change of the variable of integration $t-t^{\prime}=s$ and use the fact that $\dot{\gamma}(t)=\dot{\gamma} H(-t)$, with $H$ the Heaviside step function:

$$
P_{y x}(t)=-\dot{\gamma} \int_{t}^{\infty} G(s) d s .
$$

Note that this theory applies only in the range of linear stress responses, such as the shear-stress response to a small shear rate or the normal stress response in the case of elongational flows or combinations of shear and elongational flows with small deformation rates. Normal stress differences in shear flow, however, are a nonlinear effect and thus not captured by this linear rheological equation of state. The relaxation of normal stresses and normal stress differences are briefly discussed in Sec. IV.

\section{SIMULATION DETAILS}

We simulate an atomic fluid whose interactions are mediated via a Weeks-Chandler-Andersen (WCA) [41] potential and the equations of motion are integrated with the Gear predictor-corrector algorithm with a time step of $\Delta t=0.001$ in reduced units. All physical quantities presented are reduced using the particle mass $m$, interaction length scale $\sigma$, and the potential energy well depth $\epsilon$. These scales are set to unity in the simulations. All quantities are reduced using the LennardJones parameters and the mass; length $\mathbf{r}_{i j}^{*}=\mathbf{r}_{i j} / \sigma$, number density $\rho^{*}=\rho \sigma^{3} / m$, temperature $T^{*}=k_{\mathrm{B}} T / \epsilon$, pressure ten- sor $\mathbf{P}^{*}=\mathbf{P} \sigma^{3} / \epsilon$, strain rate $\dot{\gamma}^{*}=\dot{\gamma}\left(m \sigma^{2} / \epsilon\right)^{1 / 2}$, and viscosity $\eta^{*}=\eta \sigma^{2}(m \epsilon)^{-1 / 2}$. All presented quantities are reduced and the asterisk notation will be dropped henceforth.

The equilibrium simulations in this work start from a lattice containing 4000 atoms. The fluid is equilibrated until a steady-state is reached. After the equilibration, the calculation of correlation functions is started, with a maximum lag time $t=10$. We average the correlation function over $6.0 \times 10^{7}$ time steps to gather enough accumulations. Each simulation in this work is performed at a temperature $T=1.0$. A Gaussian isokinetic thermostat [42] is used to control the temperature of the fluid.

Our relaxation simulations are performed at a density $\rho=0.84$ and temperature $T=1.0$. The fluid is sheared at a constant rate until a steady state is reached, after which point the driving field is suddenly removed. The nonequilibrium simulations contain 512 atoms and the data are obtained from an average over $12 \times 10^{4}$ trajectories. To maintain a constant temperature, the generated heat needs to be removed from the system. A configurational thermostat $[43,44]$ is used to control the temperature during the relaxation to equilibrium.

\section{RESULTS AND DISCUSSION}

The infinite-frequency shear modulus, zero-shear normal stress coefficient, and relaxation time are calculated from the equilibrium stress autocorrelation function, by applying the theory presented in Sec. II to our MD simulations.

Figure 1 shows the zero-shear viscosity and zero-shear first normal stress coefficient for a fluid in equilibrium at a density $\rho=0.84$ and temperature $T=1.0$. Comparing both stress relaxation functions illustrates the large amount of averaging needed for accurate calculations of the zero shear rate normal stress coefficient. The zero-shear normal stress coefficient converges to a value within error bars of the value calculated using the same method by Daivis et al. [22] ( $\Psi_{1,0}=0.40 \pm 0.02$ ) but higher than the values obtained from extrapolating NEMD results. This discrepancy could be related to an unknown shear rate dependence of the normal stress coefficient at extremely low shear rate. However, it seems more likely that the action of the homogeneous thermostat in NEMD simulations is responsible for a discontinuous change in the first normal stress coefficient from the equilibrium value. As discussed in Ref. [22], there may not be a unique thermodynamic temperature for thermostatted NEMD systems, and the calculation of second-order properties are sensitive to the thermostatting mechanism used, because the normal stress differences and the nonequilibrium corrections to the temperature are both of $O\left(\dot{\gamma}^{2}\right)$.

The relaxation time can be calculated using Eq. (11). Since the integral of the shear relaxation modulus quickly converges to a constant value, the relaxation time (not shown here) converges in a similar way to the zero shear rate first normal stress coefficient, shown in Fig. 1(b). The viscous relaxation time obtained at the current state point is $0.098 \pm 0.002$.

The stress relaxation modulus for a WCA fluid at densities $\rho=0.08,0.12,0.20,0.28,0.36,0.44,0.52,0.60,0.68$, $0.76,0.84$. 0.88, and 0.92 is shown in Fig. 2. All of the curves show the same behavior at small times. The relaxation modulus has a zero slope at $t=0$, which is directly followed 


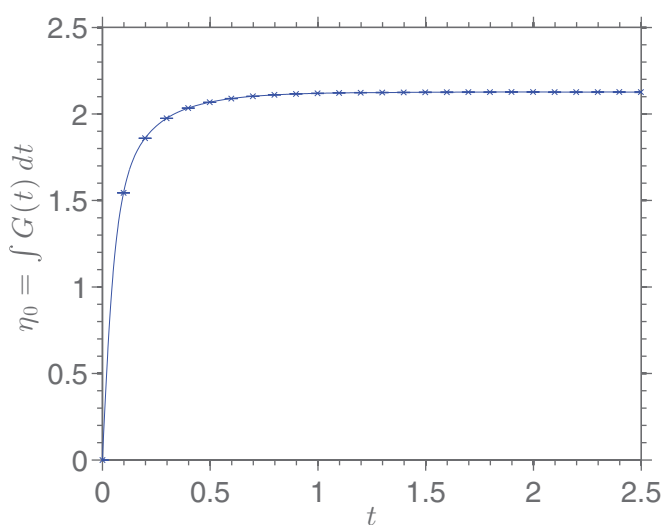

(a)

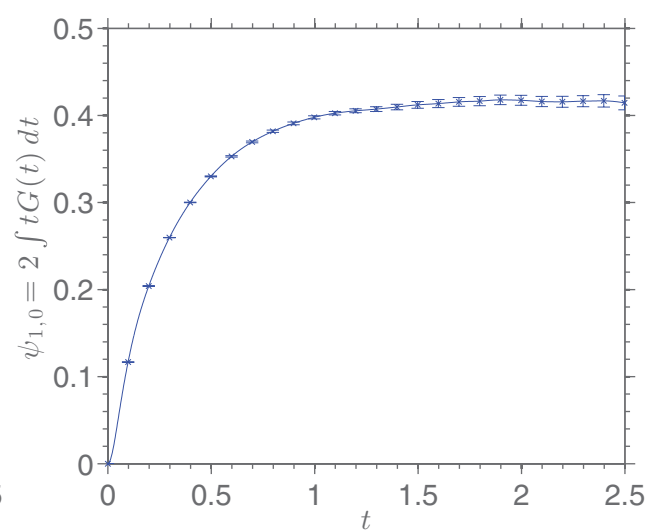

(b)

FIG. 1. (Color online) Shear viscosity and normal stress coefficient. The data are averaged over $6.0 \times 10^{7}$ time steps. The fluid has a density $\rho=0.84$ and temperature $T=1.0$.

by a Gaussian-like fast decay. This can be understood by considering a power series expansion of the autocorrelation function $C_{P P}(t)=\left\langle\mathbf{P}^{0 s}(t): \mathbf{P}^{0 s}(0)\right\rangle$ around $t=0$ [45]:

$$
\begin{aligned}
C_{P P}(t)= & C_{P P}(0)-\frac{t^{2}}{2 !}\left\langle\dot{\mathbf{P}}^{0 s}(0): \dot{\mathbf{P}}^{0 s}(0)\right\rangle \\
& +\frac{t^{4}}{4 !}\left\langle\ddot{\mathbf{P}}^{0 s}(0): \ddot{\mathbf{P}}^{0 s}(0)\right\rangle+O\left(t^{6}\right) .
\end{aligned}
$$

This expansion has a functional form similar to that of a power series expansion of a Gaussian function.

At longer times, the decay of the kernel depends strongly on the density. The rate of decay of the kernel decreases with the density at low densities $\rho \leqslant 0.68$, whereas the opposite trend is observed at higher densities $\rho>0.68$. The different behavior of the correlation function at high densities is due to the long-time tail that is observed in the configurational part of the SACF near the solid-liquid transition.

In the dilute limit, the shape of the shear modulus can be accurately approximated with the sum of a Gaussian plus an exponential decay:

$$
G(t) \approx G^{\infty}\left(\mathrm{Ae}^{-t^{2} /\left(2 \tau_{1}^{2}\right)}+(1-A) e^{-t / \tau_{2}}\right),
$$

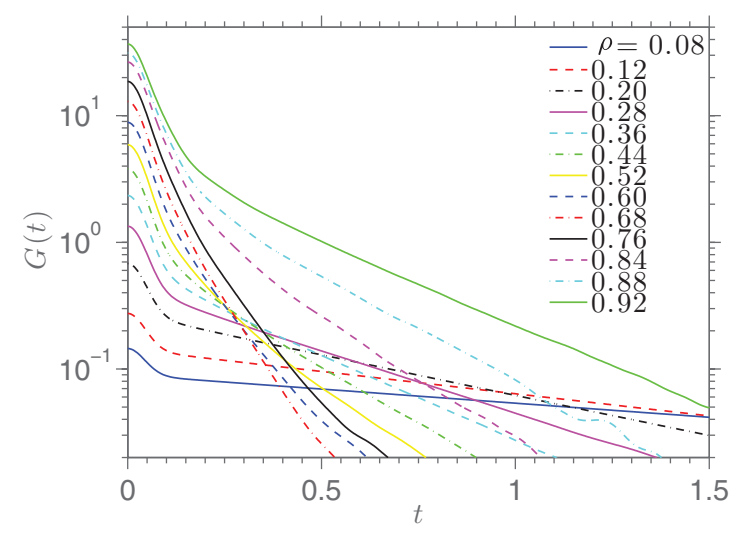

FIG. 2. (Color online) The stress relaxation modulus at different densities and a temperature $T=1.0$. Each stress autocorrelation function is averaged over $6.0 \times 10^{7}$ time steps. $G(0)$ increases monotonically with increasing density. where $A$ is a measure of the initial relative magnitude of the Gaussian mode of relaxation, $\tau_{1}^{2}$ is the variance of the Gaussian, and $\tau_{2}$ is the relaxation time of the exponentially decaying mode. Powles and Heyes [3] suggested a fitting function, for the shear stress and pressure autocorrelation functions, of the form $C(t) \propto \operatorname{sech}(\sqrt{2 T} n t)$, where $n$ is the power of the repulsive potential. Since this is an even function in time, it satisfies the form of the Taylor series expansion around $t=0$, given in Eq. (15). Furthermore, at long times, this function approaches an exponential decay, such as predicted by the Maxwell model. Brańka and Heyes [4] showed later, for the pressure autocorrelation function, that it is not possible to represent the steeply repulsive behavior of the relaxation function by the suggested functional form, and an additional singular function is required. This makes their fitting function more complicated than the functional form suggested in Eq. (16).

Least-squares fits of the shear moduli of a fluid of densities $\rho=0.08,0.12,0.20$, and 0.28 are shown in Fig. 3(a) and the corresponding fitting parameters are given in Table I. The values for $A$ show an increasing trend with density. This trend implies that only in the dilute limit will a single exponential (which is, for example, assumed in a Maxwell model) become a reasonable approximation of the stress relaxation modulus. The variance of the Gaussian $\tau_{1}^{2}$ shows no strong density dependence, even for higher densities. We observe that the fitted relaxation times of the exponential mode $\tau_{2}$ decrease with increasing density (for dilute fluids). Since the contribution of the Gaussian decays quickly to zero, the exponential mode gives a good approximation of the relaxation time of the stress relaxation modulus at low densities. As the density increases to $\rho>0.28$, the functional form of the shear modulus is no longer well described by Eq. (16). At higher densities $(\rho \geqslant 0.68)$, the shape of the kernel becomes more difficult to describe. The increasing trend in $A$ at low densities does not continue at higher densities. Instead, the relative magnitude of the first mode decreases at high density, while the variance of the Gaussian mode shows very little dependence on the density. A long-time tail forms at high densities, which can be assumed to be an additional mode of relaxation. Fits of the high-density relaxation modulus are shown in Fig. 3(b). The data, from top 


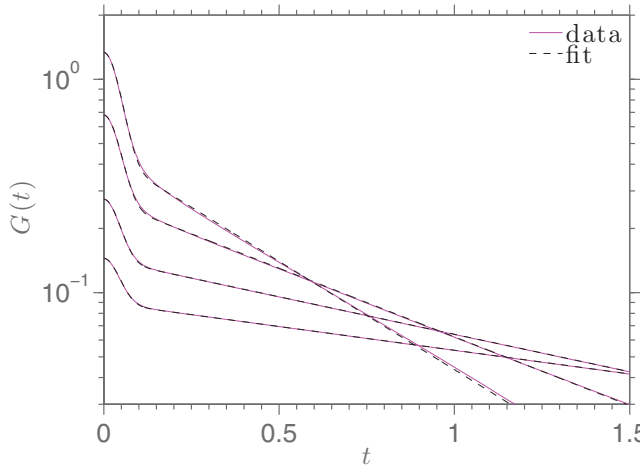

(a)

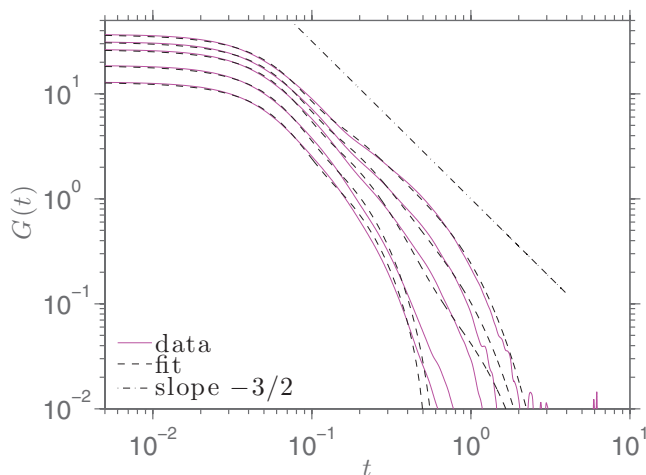

(b)

FIG. 3. (Color online) The shear modulus of a dilute (a) and dense (b) fluid. The dilute fluid is fitted with the sum of a Gaussian and an exponential; the curves correspond from bottom to top (left of the crossings) to $\rho=0.08,0.12,0.20$, and 0.28 . The relaxation functions of dense fluids are fitted with the sum of a Gaussian and two exponentially decaying modes. The curves correspond from bottom to top to $\rho=0.68,0.76,0.84,0.88$, and 0.92 .

to bottom, correspond to densities $\rho=0.92,0.88,0.84,0.76$, and 0.68 , respectively. The functional form of the fit is given by the the sum of a Gaussian and two exponential modes:

$$
G(t) \approx G^{\infty}\left(\mathrm{Ae}^{-t^{2} /\left(2 \tau_{1}^{2}\right)}+\mathrm{Be}^{-t / \tau_{2}}+(1-A-B) e^{-t / \tau_{3}}\right) .
$$

This functional form results in reasonable fits of the data corresponding to the highest densities ( $\rho=0.88$ and 0.92 ), but is less successful when applied to the data for $\rho=0.84$. Furthermore, neither of the relaxation functions shows a tail that is proportional to $t^{-3 / 2}$ over a large time range. However, the relaxation function corresponding to $\rho=0.84$ shows an exponent that is close to $-3 / 2$ over a short range of time.

The integral of the shear relaxation modulus results in the zero shear rate viscosity [Eq. (10)]. Figure 4 shows the shear viscosities corresponding to the shear moduli shown in Fig. 2. The data show a similar trend to the simulation results from Silva et al. [46] for a WCA fluid and the data from Baidakov et al. [47] and Todd [48] for a Lennard-Jones fluid. The latter show quantitative differences in the dilute and dense limits. This disagreement is due to the different interaction potential, as illustrated by the results of Cappelezzo et al. [49]. They used a Stokes-Einstein relation to calculate the shear viscosity of simple fluids at equilibrium. Their data, for a WCA fluid

TABLE I. Fitting parameters for the dilute and dense stress relaxation moduli.

\begin{tabular}{lccccc}
\hline \hline$\rho$ & $A$ & $B$ & $\tau_{1}^{2}$ & $\tau_{2}$ & $\tau_{3}$ \\
\hline 0.08 & 0.3814 & & 0.0016 & 1.9538 & \\
0.12 & 0.4778 & & 0.0016 & 1.2362 & \\
0.20 & 0.5986 & & 0.0016 & 0.6746 & \\
0.28 & 0.6635 & & 0.0017 & 0.4281 & \\
0.68 & 0.6218 & 0.8781 & 0.0018 & 0.1270 & 0.1465 \\
0.76 & 0.6050 & 0.7597 & 0.0018 & 0.1197 & 0.1396 \\
0.84 & 0.5890 & 0.3978 & 0.0019 & 0.1051 & 0.4666 \\
0.88 & 0.5785 & 0.3738 & 0.0019 & 0.1016 & 0.3723 \\
0.92 & 0.5664 & 0.3471 & 0.0019 & 0.0976 & 0.3882 \\
\hline \hline
\end{tabular}

at density $\rho=0.85$ and temperature $T=1.0$, agree well with the viscosity of our WCA fluid around that density and the Lennard-Jones fluid shows good agreement with the viscosity of the Lennard-Jones fluid reported by Baidakov et al. [50]. Furthermore, the authors reported viscosities of a LennardJones fluid and a WCA fluid at a temperature $T=1.5$ over a range of densities. The discrepancy between viscosities of WCA and LJ fluids at this temperature was consistent with the behavior in Fig. 4; at low densities the WCA fluid has a higher viscosity than the Lennard-Jones fluid, but at high densities it is the other way around. Silva et al. [46] proposed an expression to relate the viscosity of a dense WCA fluid $(\rho \geqslant 0.6)$ to that of a Lennard-Jones fluid at the same state point. This relation is given by

$$
\frac{\eta_{\mathrm{LJ}}}{\eta_{\mathrm{WCA}}}=\theta_{0}+\frac{\theta_{1}}{T},
$$

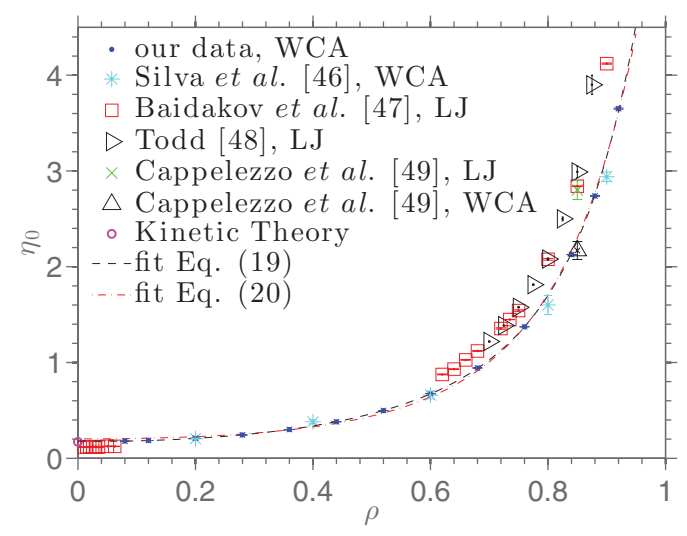

FIG. 4. (Color online) The zero-shear viscosity as a function of density, obtained from EMD simulations at a temperature $T=1.0$. The data are compared to the values reported by Silva et al. [46] for a WCA fluid, the data from Baidakov et al. [47] and Todd [48] for a Lennard-Jones fluid, and data from Cappelezzo et al. [49] for Lennard-Jones and WCA fluids. Furthermore, our data are fitted with two functions and the zero-density shear viscosity is predicted with kinetic theory. 
where $\theta_{0}$ and $\theta_{1}$ are fitting parameters. The authors found $\theta_{0}=0.9932$ and $\theta_{1}=0.2778$, which means that for a fluid at temperature $T=1.0$ we find $\eta_{\mathrm{LJ}}=1.271 \eta_{\mathrm{WCA}}$ for all densities $\rho \geqslant 0.6$. This expression gives a reasonable estimate, but in practice the ratio between both viscosities depends on the density as well as the temperature, as shown by the data listed by Silva et al. [46]. This scaling results in good agreement (not shown here) between the Lennard-Jones and the WCA data in Fig. 4.

Rowley and Painter [51] presented a function to describe the dependence of shear viscosity on density and temperature. Since we only consider a single temperature here, we can simplify the model to

$$
\eta(\rho)=\eta(0) \exp \left(\sum_{i=1}^{d} C_{i} \rho^{i}\right),
$$

where $d$ is the degree of the polynomial. The fit, shown in Fig. 4, shows good agreement for $d=4$. The parameters of the fit are $\eta(0)=0.1797, C_{1}=-0.6175, C_{2}=8.2438, C_{3}=$ -8.8141 , and $C_{4}=4.8280$. The fit could be simplified further to a stretched exponential fit [48]. The stretched exponential function is given by

$$
\eta(\rho)=\eta(0) \exp \left(C_{1} \rho^{C_{2}}\right) .
$$

The model parameters are fitted as $\eta(0)=0.2019, C_{1}=$ 3.4478 , and $C_{2}=2.1433$. This functional form leads to a less accurate fit than Eq. (19) with $d=4$, especially in the dilute region.

The zero-density limit viscosity $\eta(0)=0.1797$ that we found from the most accurate fit [Eq. (19)] can be compared to the predicted value from kinetic theory for the zero-density limit. The theory predicts a zero-shear viscosity given by [19]

$$
\eta_{0}=\frac{p}{v}
$$

where $p$ is the hydrostatic pressure and $v$ the collision rate. The pressure of a dilute fluid is given (in physical units) by

$$
p=\rho k_{\mathrm{B}} T
$$

where $\rho$ and $T$ are the number density and temperature, respectively. The collision rate $v$ can be expressed in terms of the Lennard-Jones parameters

$$
v=3 \rho A_{2}, \quad A_{2}=1.3703 \sqrt{\frac{2 \epsilon}{m}}=1.938,
$$

where the numerical value for $A_{2}$ corresponds to Maxwell molecules [52], which are softer than WCA atoms. However, as discussed in Ref. [19], the collision rate for dilute fluids shows little dependence on the interaction potential used. Substituting the parameters for our simulations, we find a viscosity $\eta(0)=k_{\mathrm{B}} T\left(3 A_{2}\right)^{-1}=0.1720$. This result is within $5 \%$ of the value that we found from the fit of the MD simulation results. Furthermore, Rowley and Painter [51] have presented an expression, based on the Chapman-Enskog theory, for the zero-density shear viscosity of a Lennard-Jones fluid. Using their expression for a fluid at temperature $T=1.0$, the zero-density shear viscosity is $\eta(0)=0.1256$. This value is much lower than the WCA data suggest, but more consistent with the Lennard-Jones data from Baidakov et al. [47].

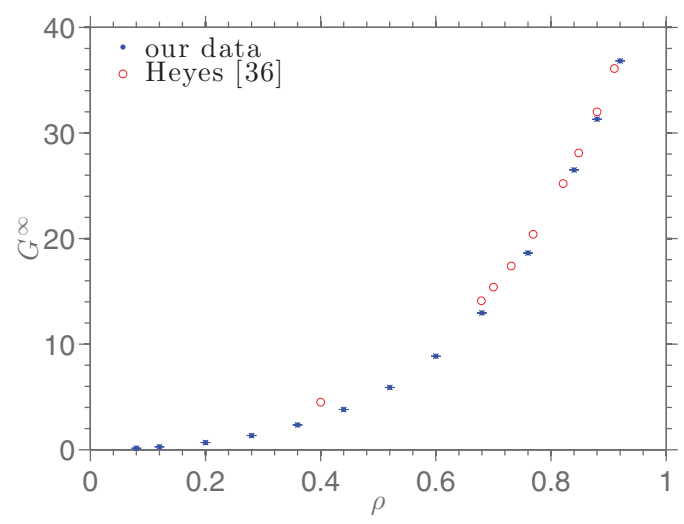

FIG. 5. (Color online) The infinite-frequency shear modulus as a function of density, obtained from EMD simulations at a temperature $T=1.0$. The data are compared to the values reported by Heyes [36] for a Lennard-Jones fluid at a temperature $T=1.06$.

The infinite-frequency shear modulus, as a function of density, is shown in Fig. 5. The interaction potential used to model the fluid has a strong influence on the value of the infinite-frequency shear modulus, as mentioned in Sec. II. In the hard-sphere limit, the modulus goes to infinity for soft-sphere potentials, as can be understood from the integral relation given by Zwanzig and Mountain [13]. The nonlinear relation between pressure and density leads to a strong increase in $G^{\infty}$ with increasing density. The modulus approaches zero in the low-density limit, which means that the elastic response becomes negligibly small and the fluid has no notable memory. The infinite-frequency shear moduli for densities $\rho=0.68$, 0.78 , and 0.88 are compared to the values reported by Heyes [36] for a Lennard-Jones fluid at a temperature $T=1.06$. Good agreement is found for these values, although an exact quantitative comparison is not possible since the author used a different interaction potential and the temperature is slightly different. However, we note that the Lennard-Jones and WCA potentials have the same power in the repulsive part. Furthermore, the shear modulus is known to be only moderately dependent on temperature [53].

Figure 6 shows the relaxation times, calculated according to Eq. (11), as well as the Maxwell relaxation time, as a function of the density. Both measures of relaxation time show the same qualitative trend. In the dilute regime, the relaxation time

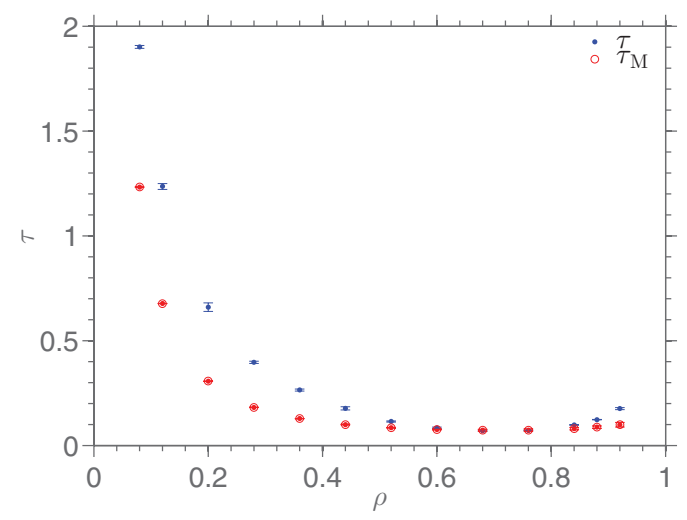

FIG. 6. (Color online) Relaxation time as a function of density, compared to the Maxwell relaxation time. 


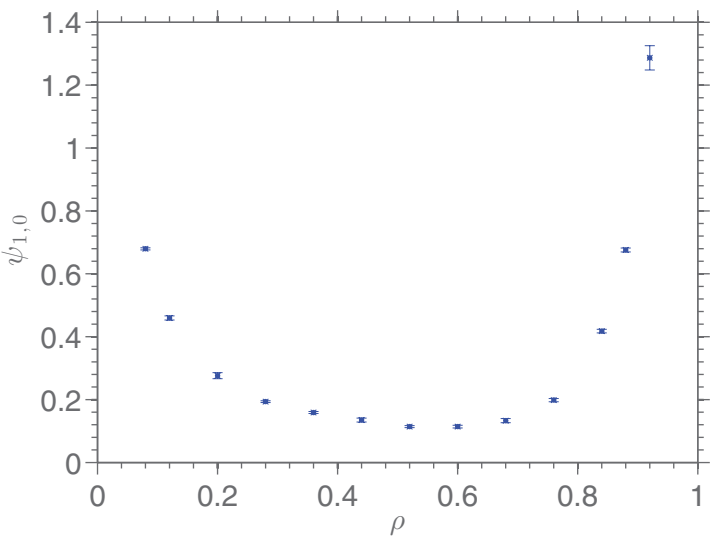

FIG. 7. (Color online) First normal stress coefficient as a function of density, obtained from EMD simulations.

clearly decreases with an increasing density. The low-density relaxation times are in good agreement with the relaxation time of the exponential mode, shown in Table I. The relaxation times reach a minimum towards the dense regime $(\tau=0.071$ and $\tau_{M}=0.073$ at $\rho=0.68$ ) and then increase with density in the dense regime, due to the formation of the molasses tail.

Figure 7 shows the zero-shear first normal stress coefficient as a function of the density. The normal stress coefficient and the slope of the function increase towards the dense and the dilute extremes, while a minimum is found around $\rho=0.52$. This remarkable trend was not observed when $\Psi_{1,0}$ was found by extrapolating nonequilibrium simulation data [14].

The larger normal stress coefficients at high densities are related to the memory effect of the viscoelastic fluid. The trend of the zero-shear normal stress differences in the dilute regime can be compared with the prediction from kinetic theory, in a way similar to that done for the viscosity [Eq. (21)]:

$$
\Psi_{1,0}(\rho)=\frac{2 p}{v^{2}}=\frac{2 k_{\mathrm{B}} T}{9 \rho A_{2}^{2}} .
$$

This prediction from kinetic theory shows good agreement with our simulation data for densities $\rho \leqslant 0.52$, as shown in Fig. 8. The slight overprediction can be understood since the Maxwell molecules are softer than the WCA atoms. The harder

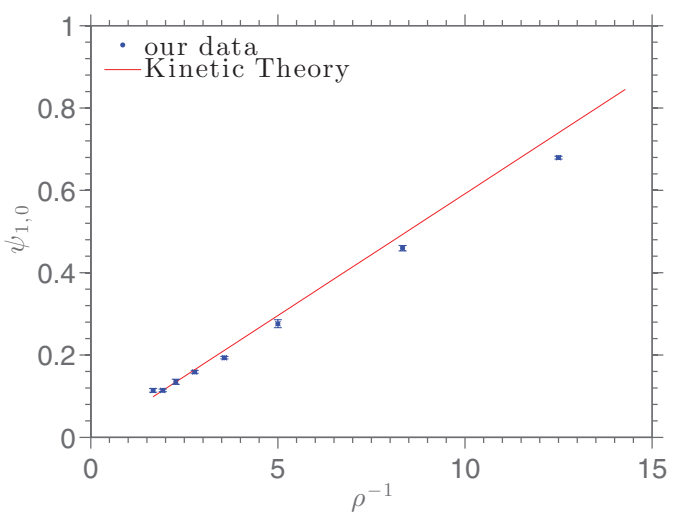

FIG. 8. (Color online) First normal stress coefficients of a dilute fluid, compared to kinetic theory.

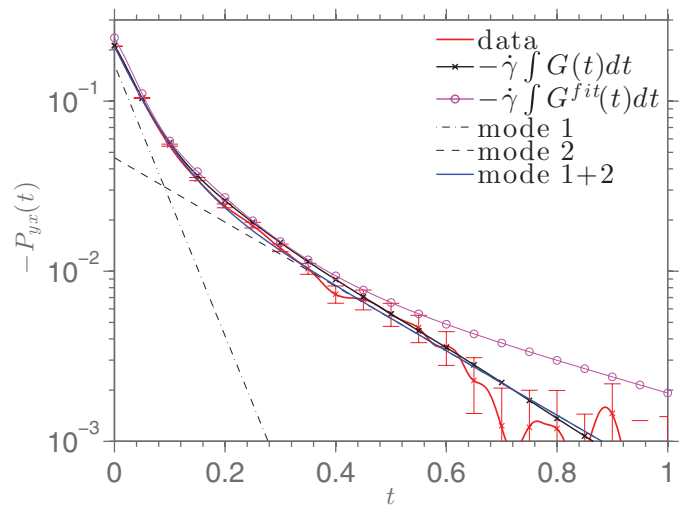

FIG. 9. (Color online) In the linear regime $(\dot{\gamma}=0.1$ shown), the relaxation of shear stress after cessation of steady shear can be accurately predicted from equilibrium statistical mechanics. The sum of two exponential relaxation modes also fit the data well $(\rho=0.84$, $T=1.0$ ). The fitting parameters are $C=-0.8041, \tau_{1}=0.0565$, and $\tau_{2}=0.2482$.

WCA potential leads to a slightly larger collision rate, which results in a lower normal stress coefficient.

We have already shown that at moderate density, the stress relaxation function is well described by a Gaussian plus two exponentials. This can be integrated to find the stress response after the cessation of steady shear flow using Eq. (14). A similar approach is used by Picu and Weiner [8] to approximate the stress relaxation of a viscoelastic fluid

$$
P_{\alpha \beta}(t)=\sum_{i} C_{i} e^{-t / \tau_{i}}
$$

where each stress component $P_{\alpha \beta}$ can have different proportionality constants $C_{i}$ and relaxation times $\tau_{i}$. The shear stress relaxation of a simple fluid can be approximated with two modes [38]. Thus, 4 unknowns $\left(C_{1}, \tau_{1}, C_{2}, \tau_{2}\right)$ have to be found to fit the simulation data to a function of the form of Eq. (25). We can reduce the number of fitting parameters for the shear stress by substituting $C_{2}=P_{y x}(0)-C_{1}$. Taking $P_{y x}(0)$ in front of the equation and dropping the subscript of the constant reads

$$
P_{y x}(t)=P_{y x}(0)\left(C e^{-t / \tau_{1}}+(1-C) e^{-t / \tau_{2}}\right) .
$$

The constant $C$ represents the initial weight of the first mode relative to the second.

In Fig. 9, the relaxation after a sudden cessation of steady shear flow for $\dot{\gamma}=0.1$ is shown for a dense liquid at $\rho=0.84$ and temperature $T=1.0$. The relaxation of shear stress obtained from a NEMD simulation is in good agreement with the relaxation modulus predicted from linear viscoelastic theory. This confirms that the linear viscoelastic theory, presented in Eq. (14), is satisfied. The shear stress is also predicted by substituting the fit of the shear modulus of a dense fluid [Eq. (17)] into Eq. (14), with the fitting parameters reported in Table I. This integrated fit shows good agreement with the shear stress at short times, but deviates at longer times. This may be expected since the fit of the shear modulus [Fig. 3(b)] showed a slower decay than the data in this region, which correspond to an overprediction of the shear stress. Furthermore, both modes of the double 
exponential fit [Eq. (26)], and the combination of the modes is shown in the figure. The fit is shown to be in good agreement with the shear stress data, with fitting parameters $C=-0.8041, \tau_{1}=0.0565$, and $\tau_{2}=0.2482$. However, two exponential relaxation functions are not enough to capture all the mechanisms related to structural relaxation of a dense liquid, since we have seen that the stress relaxation modulus shows an initial Gaussian-like shape (Fig. 2), followed by multiple relaxation modes. Especially at very short times, the slope of the fit deviates from that of the stress relaxation function, while the function calculated with Eq. (14) is in perfect agreement.

The relaxation time obtained from Eq. $(11)(\tau=0.098)$ lies in between the relaxation times of the two fitted exponential modes. This is because the overall relaxation time is a weighted average of the spectrum of relaxation modes present in the fluid. An example to illustrate this point can be given by taking the time derivative of Eq. (26) and defining a time scale as $\tau_{*}^{-1}=\dot{P}_{y x}(0) / P_{y x}(0)$ :

$$
\frac{\dot{P}_{y x}(0)}{P_{y x}(0)}=\frac{1}{\tau_{*}}=\left(\frac{C}{\tau_{1}}+\frac{1-C}{\tau_{2}}\right) .
$$

This results in a different time scale $\left(\tau_{*}=0.067\right)$ than defined by Eq. (11). We know that if linear viscoelastic theory is valid $\dot{P}_{y x}(0)=\dot{\gamma} G^{\infty}$ and $P_{y x}(0)=\dot{\gamma} \eta$, where $\eta$ is close to the Newtonian viscosity. For small enough shear rates, such that the stress response is a linear function of the shear rate, the time scale defined in Eq. (27) becomes $\tau_{*}=\eta / G^{\infty}$. This expression is equal to the Maxwell relaxation time that is often used as the characteristic time in the linear viscoelastic Maxwell model [54]. This model is based on the assumption that the relaxation function can be accurately described with a single exponential. We have shown that this is not the case for any of the fluid densities studied here.

We now briefly discuss the stress relaxation from a steady shear flow at a shear rate $\dot{\gamma}=1.0$. At high shear rates, the shear stress is not linearly proportional to the strain rate and linear viscoelastic theory is expected to break down. Non-Newtonian phenomena, such as shear thinning, shear dilatancy, and normal stress differences, are known to occur for shear rates of this magnitude. Figure 10 confirms that the linear viscoelastic theory is not a good approximation at this high shear rate. The shear stress relaxes faster than linear viscoelastic theory predicts. Furthermore, the linear theory does not account for the shear thinning that is responsible for the deviation between the data and the EMD prediction for the shear stress at $t=0$ (the steady-state shear stress before relaxation takes place). The double exponential fit, with fitting parameters $C=-0.9452, \tau_{1}=0.0473$, and $\tau_{2}=0.2271$, is still a good approximation of the shape. The second relaxation time $\tau_{2}$ is almost equal at both shear rates, while the first relaxation time $\tau_{1}$ is smaller at the high shear rate, corresponding to a faster stress decay. Furthermore, the first mode is initially more dominant at high shear rate compared to the lower shear rate, i.e., larger value for $C$.

Figure 11 shows the relaxation of the normal stresses after cessation of steady shear flow at strain rate $\dot{\gamma}=1.0$. Each of the normal stress components [and thus also the hydrostatic pressure, defined as $\left.p=\frac{1}{3} \operatorname{tr}(\mathbf{P})\right]$ decays as the

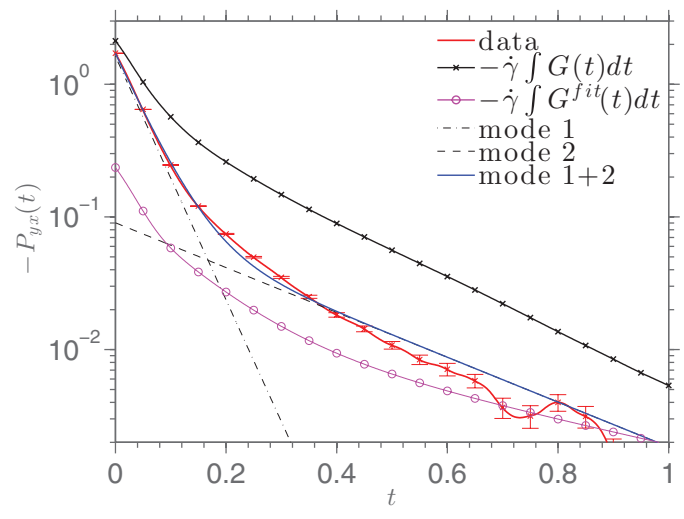

FIG. 10. (Color online) Shear stress relaxing from steady shear flow at $\dot{\gamma}=1.0$. The data are fitted with a double exponential curve. The fitting parameters are $C=-0.9452, \tau_{1}=0.0473$, and $\tau_{2}=0.2271$.

fluid relaxes to equilibrium. This demonstrates the existence of stress dilatancy in the system. Furthermore, the normal stresses are not equal to each other at $t=0$, which shows the presence of normal stress differences (shown in the inset). The relaxation of the normal stress components is different from the relaxation of shear stress. They converge to equilibrium at a lower rate than the shear stress. Furthermore, the normal stresses converge to each other much faster than they relax to equilibrium, as shown in the inset. The relaxation of the normal stress components is fitted with the sum of two exponential modes. The differences between the fits of normal stress components agree well with the relaxation of the normal stress differences. We have observed that these nonlinear transient effects behave very differently in startup flow, compared to nondriven relaxation from a nonequilibrium steady state. The stress response in a startup flow is not shown here; however, for example in Ref. [1], transient normal stress differences are shown for atomic shear flow simulations $(\dot{\gamma}=1.0)$, performed at the same state point as our simulations.

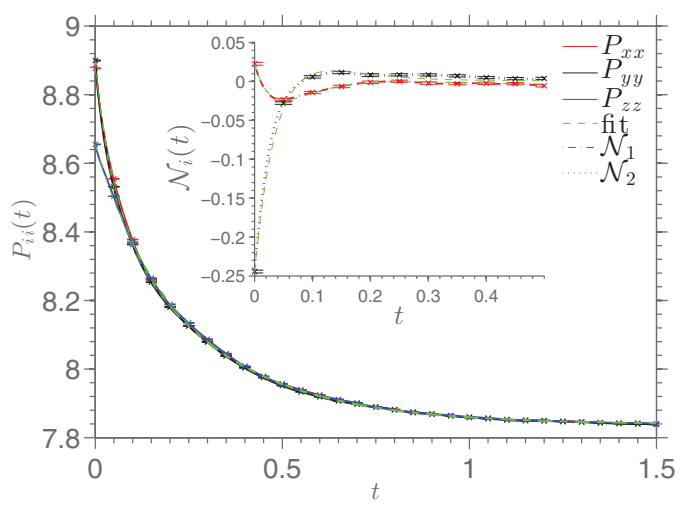

FIG. 11. (Color online) Relaxation of the diagonal components of the pressure tensor and the normal stress differences after a sudden cessation of steady shear flow $(\dot{\gamma}=1.0)$. At $t=0, P_{x x}$ and $P_{y y}$ are almost identical, while $P_{z z}$ is significantly lower. The relaxation of the normal stresses are fitted with the sum of two exponentials. The normal stress differences are shown in the inset. 


\section{CONCLUSIONS}

We have presented very accurate calculations of the shear stress relaxation modulus of a simple atomic fluid over a wide range of densities. The zero-shear viscosity, zero-shear first normal stress coefficient, and relaxation time are calculated from the appropriate integrals over the stress autocorrelation function. The accuracy of our data enables us to study the shape of the relaxation function and the density dependence of the material functions, and to make a quantitative comparison between molecular dynamics simulation results and kinetic theory.

Little is known about the functional form of the relaxation modulus. We have shown that the shear modulus of a dilute simple fluid can be accurately fitted with the sum of a Gaussian and an exponential. The width of the Gaussian shows very little variation with density, whereas the decay rate of the exponential term increases with density in the dilute regime. This leads to a decrease of the relaxation times for densities up to $\rho=0.68$. At higher densities, the behavior becomes more complicated, due to the development of a slowly decaying tail in the shear modulus. This tail leads to an increase of the relaxation time with density.

We have shown that the zero-shear viscosity and the infinite-frequency shear modulus increase monotonically with density. Both density dependencies show good agreement with data from the literature. Furthermore, the viscosity in the dilute limit agrees well with the predicted value from kinetic theory.

The zero-shear first normal stress coefficient shows a qualitatively similar density dependence to the relaxation times. The zero-shear first normal stress coefficient decreases with increasing density at low densities and strongly increases with density in the dense regime, while a minimum normal stress coefficient is found around $\rho=0.52$. The decaying trend in the dilute regime is in good agreement with the prediction from kinetic theory. This agreement is a confirmation of the validity of our MD simulations as well as the theory presented by Coleman and Markovitz [20]. In the dense region, the viscoelastic memory effect causes an increase in the normal stress coefficient towards the solid-liquid transition density.

Linear viscoelastic theory has been applied to predict the linear relaxation of the stress from a steady-state shear flow. The prediction made from equilibrium molecular dynamics results has been compared to MD simulations of a fluid relaxing from a nonequilibrium steady state. Good agreement was found for a fluid relaxing from a steady flow at shear rate $\dot{\gamma}=0.1$, whereas for a shear rate $\dot{\gamma}=1.0$ the shear stress relaxes faster than the linear theory predicts. Furthermore, the shear thinning that occurs at a large shear rate is not accounted for in the linear viscoelastic theory. At both shear rates, the shear stress can be fitted reasonably well with the sum of two exponential modes. However, the shape of the relaxation modulus suggests that the double exponential fit does not capture the stress relaxation correctly at short times. A more accurate fit was achieved by accounting for the Gaussian shape at short times and the tail at longer times.

While the present study is limited to atomic fluids, extending it to molecular fluids could increase our understanding of the relaxation behavior of fluids that are more interesting from an industrial or biological point of view. However, accurate calculations of the normal stress coefficient for simple fluids already require a large amount of computation. Due to the definition of the normal stress coefficient, the computation required increases dramatically with the maximum lag time for the SACF to converge to zero.

\section{ACKNOWLEDGMENTS}

R.H. would like to acknowledge the support of Professor Stefan Luding and the financial support of NWO-STW VICI Grant No. 10828 and MicroNed Grant No. 4-A-2.
[1] D. J. Evans and G. P. Morriss, Statistical Mechanics of Nonequilibrium Liquids, 2nd ed. (Cambridge University Press, Cambridge, 2008).

[2] M. Isobe and B. J. Alder, Mol. Phys. 107, 609 (2009).

[3] J. G. Powles and D. M. Heyes, Mol. Phys. 98, 917 (2000).

[4] A. C. Brańka and D. M. Heyes, Phys. Rev. E 69, 021202 (2004).

[5] M. S. Green, J. Chem. Phys. 22, 398 (1954).

[6] R. Kubo, J. Phys. Soc. Jpn. 12, 570 (1957).

[7] A. J. C. Ladd, W. E. Alley, and B. J. Alder, J. Stat. Phys. 48, 1147 (1987).

[8] R. C. Picu and J. H. Weiner, J. Chem. Phys. 107, 7214 (1997).

[9] R. D. Mountain and R. Zwanzig, J. Chem. Phys. 44, 2777 (1966).

[10] R. D. Mountain, J. Chem. Phys. 48, 2189 (1968).

[11] E. Keshavarzi, M. Vahedpour, S. Alavi, and B. Najafi, Int. J. Thermophys. 25, 1747 (2004).

[12] B. J. Alder, D. M. Gass, and T. E. Wainwright, J. Chem. Phys. 53, 3813 (1970).

[13] R. Zwanzig and R. D. Mountain, J. Chem. Phys. 43, 4464 (1965).

[14] P. J. Daivis, J. Non-Newtonian Fluid Mech. 152, 120 (2008).
[15] G. A. Nunez, G. S. Ribeiro, M. S. Arney, J. Feng, and D. D. Joseph, J. Rheol. 38, 1251 (1994).

[16] W. Gleile and N. Ohl, Rheol. Acta 29, 261 (1990).

[17] J. G. Nam, K. H. Ahn, S. J. Lee, and K. Hyun, J. Rheol. 54, 1243 (2010).

[18] W. Loose and S. Hess, Phys. Rev. A 37, 2099 (1988).

[19] V. Garzó and A. Santos, Kinetic Theory of Gases in Shear Flows: Nonlinear Transport (Kluwer Academic Publishers, Dordrecht, 2003).

[20] B. D. Coleman and H. Markovitz, J. Appl. Phys. 35, 1 (1964).

[21] R. B. Bird, R. C. Armstrong, and O. Hassager, Dynamics of Polymeric Liquids, Vol. 1, 2nd ed. (Wiley-Interscience, New York, 1987).

[22] P. J. Daivis, B. A. Dalton, and T. Morishita, Phys. Rev. E 86, 056707 (2012).

[23] E. Cohen, Physica A 118, 17 (1983).

[24] M. H. Ernst, E. H. Hauge, and J. M. J. van Leeuwen, J. Stat. Phys. 15, 7 (1976). 
[25] J. J. Erpenbeck and W. W. Wood, J. Stat. Phys. 24, 455 (1981).

[26] T. R. Kirkpatrick, Phys. Rev. Lett. 53, 1735 (1984).

[27] T. R. Kirkpatrick and J. C. Nieuwoudt, Phys. Rev. A 33, 2651 (1986).

[28] I. M. de Schepper, A. F. E. M. Haffmans, and H. van Beijeren, Phys. Rev. Lett. 56, 538 (1986).

[29] R. M. Puscasu, B. D. Todd, P. J. Daivis, and J. S. Hansen, J. Chem. Phys. 133, 144907 (2010).

[30] J. M. Dealy, J. Rheol. 28, 181 (1984).

[31] P. J. Daivis and D. J. Evans, J. Chem. Phys. 100, 541 (1994).

[32] U. Balucani, R. Vallauri, and T. Gaskell, Phys. Scripta T25, 290 (1989).

[33] D. M. Heyes, J. Phys.: Condensed Matter 6, 6409 (1994).

[34] S. R. Williams and D. J. Evans, J. Chem. Phys. 131, 024115 (2009).

[35] S. R. Williams and D. J. Evans, J. Chem. Phys. 131, 159902 (2009).

[36] D. M. Heyes, Phys. Rev. B 37, 5677 (1988).

[37] S. Hess and D. J. Evans, Phys. Rev. E 64, 011207 (2001).

[38] J. Gao and J. H. Weiner, Macromol. 25, 1348 (1992).

[39] D. J. Evans and G. P. Morriss, Phys. Rev. A 30, 1528 (1984).

[40] A. J. C. Ladd, Mol. Phys. 53, 459 (1984).

[41] J. D. Weeks, D. Chandler, and H. C. Andersen, J. Chem. Phys. 54, 5237 (1971).
[42] D. J. Evans, W. G. Hoover, B. H. Failor, B. Moran, and A. J. C. Ladd, Phys. Rev. A 28, 1016 (1983).

[43] C. Braga and K. P. Travis, J. Chem. Phys. 123, 134101 (2005).

[44] K. P. Travis and C. Braga, J. Chem. Phys. 128, 014111 (2008).

[45] J. P. Hansen and I. R. McDonald, The Theory of Simple Liquids, 2nd ed. (Academic Press, New York, 1986).

[46] F. Da Costa Silva, L. A. F. Coelho, F. W. Tavares, and M. J. E. M. Cardoso, Int. J. Quantum Chem. 95, 79 (2003).

[47] V. G. Baidakov, S. P. Protsenko, and Z. R. Kozlova, J. Chem. Phys. 137, 164507 (2012).

[48] B. D. Todd, Phys. Rev. E 72, 041204 (2005).

[49] M. Cappelezzo, C. A. Capellari, S. H. Pezzin, and L. A. F. Coelho, J. Chem. Phys. 126, 224516 (2007).

[50] We have used the data from Cappelezzo et al. [49] that corresponds to 4096 atoms, since this is closest to our system size.

[51] R. Rowley and M. Painter, Int. J. Thermophys. 18, 1109 (1997).

[52] C. Truesdell and R. Muncaster, Fundamentals of Maxwell's Kinetic Theory of a Simple Monatomic Gas: Treated As a Branch of Rational Mechanics (Academic Press, New York, 1980).

[53] N. B. Olsen, J. C. Dyre, and T. Christensen, Phys. Rev. Lett. 81, 1031 (1998).

[54] P. Attané, J. Pierrard, and G. Turrel, J. Non-Newtonian Fluid Mech. 18, 295 (1985). 